\title{
Vía aérea dificil: interacciones entre ortodoncia y anestesiología
}

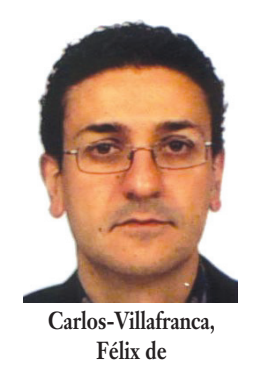

Difficult airwą: relationship between orhodontics and anaeshesiologu

\section{Carlos-Villaffance, Fêlix de* Cobo-Plana, Juan** Macías-Escalada, Enilio*** Marlínez, Juliana $a^{* * *}$}

*Profesor Asociado de Ortodoncia, Universidad de Oviedo

**Catedrático de Ortodoncia, Universidad de Oviedo

${ }^{* *}$ Colaborador de Honor, Universidad de Oviedo

$* * * *$ Postgrado de Ortodoncia. Universidad de Oviedo

\section{Correspondencia}

Félix de Carlos Villafranca

Clínica Universitaria de Odontología

Universidad de Oviedo

Catedrático Serrano s/n

33006 (Oviedo) Asturias

Email: fcarlos@uniovi.es iao@odontologico.com
Resumen: Introducción: La intubación endotraqueal es una técnica bien estandarizada pero sin embargo, dependiendo de la experiencia del operador, alteraciones anatómicas o impuestas por la patología, pueden existir situaciones en las que no se puede ventilar al paciente. Objetivo: Pretendemos identificar la implicación de nuestra disciplina en el control de la via aérea de pacientes que requieren anestesia o reanimación respiratoria o hemodinámica. Metodos: Se han estudiado los hallazgos clínicos denominados «poco tranquilizadores» publicados en la guía clínica de la Sociedad Americana de Anestesiología (ASA) para establecer una via aérea difícil a la intubación. Hemos tratado de ver y traducir estas situaciones clínicas desde el punto de vista ortodóncico-odontológico. Resultados: De los once criterios de riesgo de la ASA, podemos modificar favorablemente, con tratamientos ortodóncicos y ortopédicos convencionales, seis de ellos y parte de las situaciones adscritas al apartado cinco (grados III y IV de la clasificación de Mallampati-Samsoon). Conclusión: Parece adecuado considerar las posibilidades del tratamiento ortodóncico y la ortopedia dentofacial, no sólo como instrumento de tratamiento de maloclusiones habituales, sino como profilaxis de algunas situaciones que pueden desencadenar problemas ante situaciones de urgencia, como es la dificultad de intubación previa a una intervención quirúrgica.

Palabras clave: Vía aérea dificil, Intubación endotraqueal, Ortodoncia, Vía aérea superior.

Abstract: Introduction: Endotracheal intubation is a well standardised technique although depending on the experience of the operator, anatomical or pathology-related alterations, there may exist situations in which the patient cannot receive ventilation. Objective: We aim to identify the implication of our speciality in the control of the airway of patients requiring anaesthesia or respiratory or hemodynamic resuscitation. Methods: We have studied the clinical findings termed «non-reassuring» published in the PRACTICE GUIDELINES FOR MANAGEMENT OF THE DIFFICULT AIRWAY of the American Society of Anaesthesiology (ASA) in order to establish an airway difficult for intubation. We have attempted to view and translate these clinical situations from the orthodontic-odontologic perspective. Results: Of the eleven ASA risk criteria, we can favourably modify, with conventional orthodontic and orthopaedic treatments, six of these and part of the situations ascribed to Section five (degrees III and IV of the Mallampati-Samsoon classification). Conclusion: It seems fitting to consider the possibilities of orthodontic treatment and dentofacial orthopaedics, not only as an instrument for treating habitual malocclusions, but rather as a prophylaxis for some situations that can trigger off problems in emergency situations, such as the difficulty of intubation before a surgical operation.

Key words: Difficult airway, Endotracheal intubation, Orthodontics, Upper airway.

BIBLID [1138-123X (2005)10:2; marzo-abril 125-240]

Carlos-Villafranca F de, Cobo-Plana J, Macías-Escalada E, Martínez J. Vía aérea difícil: interacciones entre ortodoncia y anestesiología. RCOE 2005;10(1):187-192. 


\section{Introducción}

Desde hace algún tiempo, con mayor o menor asiduidad, han venido apareciendo en un gran número de revistas médicas y odontoestomatológicas, distintos artículos que estudian la efectividad, eficacia, resultados, indicaciones y contraindicaciones de los aparatos intraorales (AIO) en el tratamiento del síndrome de apneahipoapnea obstructiva del sueño (SAHS). Las relaciones con otros especialistas médicos son frecuentes en los profesionales que nos dedicamos a la ortodoncia en exclusiva (maxilofaciales, pediatras, otorrinolaringólogos [ORL]). Con la aparición de los AIO en la patología obstructiva del sueño, los ortodoncistas hemos tenido la oportunidad de relacionarnos más con ellos y a la vez establecer nuevos vínculos con otras especialidades: neumólogos, radiólogos, anestesistas,...

En esta ocasión, no pretendemos evaluar ninguno de estos aparatos ni tampoco defender las bondades de su utilización frente a otras terapias más agresivas o más costosas. Vamos a tratar de identificar la ortodoncia ante diferentes tipos de situaciones que pueden redundar en un beneficio no sólo odontológico y estético sino, por ejemplo la implicación de nuestra disciplina en el control de la vía aérea de pacientes que requieren anestesia o reanimación respiratoria o hemodinámica.

En la última reunión de consenso nacional sobre el SAHS realizada por el Grupo Español del Sueño, celebrada recientemente en Madrid, se acordó por unanimidad denominar a la aparatología intraoral para el tratamiento del SAHS, como Dispositivos de Avan- ce Mandibular (DAM, productos sanitarios a medida), término que será utilizado en toda la exposición.

\section{Metodología utilizada en la exploración de la vía aérea superior}

Si nos vamos a referir a la vía aérea superior (VAS) hemos de hacerlo en toda su extensión; por tanto, deberemos comenzar por un examen rinológico (narinas, fosas nasales, cornetes, meatos, etc.) realizado por un especialista ORL. A este nivel, las zonas en las que con más frecuencia aparecen dificultades al paso del aire son: la válvula nasal, septo y los cornetes. La válvula nasal está localizada entre el vestíbulo y las fosas nasales y en ella tiene importancia el ángulo formado por los cartílagos triangular y cuadrangular. Cuando este es inferior a $10-15^{\circ}$ se favorece el colapso por efecto Venturi ante situaciones de inspiración forzada. Esto es especialmente cierto en pacientes dolicofaciales con nariz larga y estrecha. Le siguen en frecuencia las desviaciones del septo y la hipertrofia de cornetes. Si hemos comenzado por el examen rinológico ha sido para constatar una evidencia descrita ya en 1989 por Timms y cols ${ }^{* *}$ refrendada por $\mathrm{KurO}^{2^{*}}$ en 1996. Am-bos autores describieron cómo algunos tratamientos de ortodoncia (vgr. expansión rápida palatina), consiguen dilataciones de la válvula nasal y del área turbinal inferior superiores al 33\%. Este hecho, a veces no bien conocido, puede ayudar a solucionar algunos problemas contemplados sólo desde la esfera ORL.
Completado el examen rinológico, la evaluación de la VAS continúa ahora con la exploración de la boca, lengua y orofaringe. En éstos pacientes el examen oral no sólo es importante desde el punto de vista diagnóstico, sino incluso, como marcador pronóstico ante la posible decisión terapéutica con DAM de los procesos respiratorios obstructivos.

La inspección de la lengua es uno de los métodos de valoración médica más antiguos que existen, aunque hoy ha ido perdiendo protagonismo quizá ante el amplio abanico de posibilidades diagnósticas que hoy tenemos a nuestro alcance. Estimaremos su estado, posición y tamaño. Aunque los dos primeros se realizan sin problemas, el tamaño lingual resulta difícil de definir puesto que macroglosia es un término proporcional que relaciona un continente con el contenido. Existen algunas entidades que cursan con macroglosia franca (Down, Melkerson-Rhosenthal, mucopolisacaridosis,...), pero la controversia aparece cuando nos encontramos ante un compromiso de la VAS y una lengua de tamaño normal. Esto puede atribuirse a dos supuestos: primero que la lengua esté encerrada en una caja que ha disminuido su tamaño, como sucede tras algunos tratamientos de ortodoncia planificados con extracciones que reducen significativamente las dimensiones del continente comprometiendo el «espacio vital» lingual. Y segundo porque la lengua se encuentre desplazada por procesos de vecindad (vgr. hipertrofia amigdalar, inflamaciones,...), ante cambios en la posición de la mandíbula (vgr. dolicofaciales con respiración oral) y en algunas maloclusiones (vgr. Clase III). 

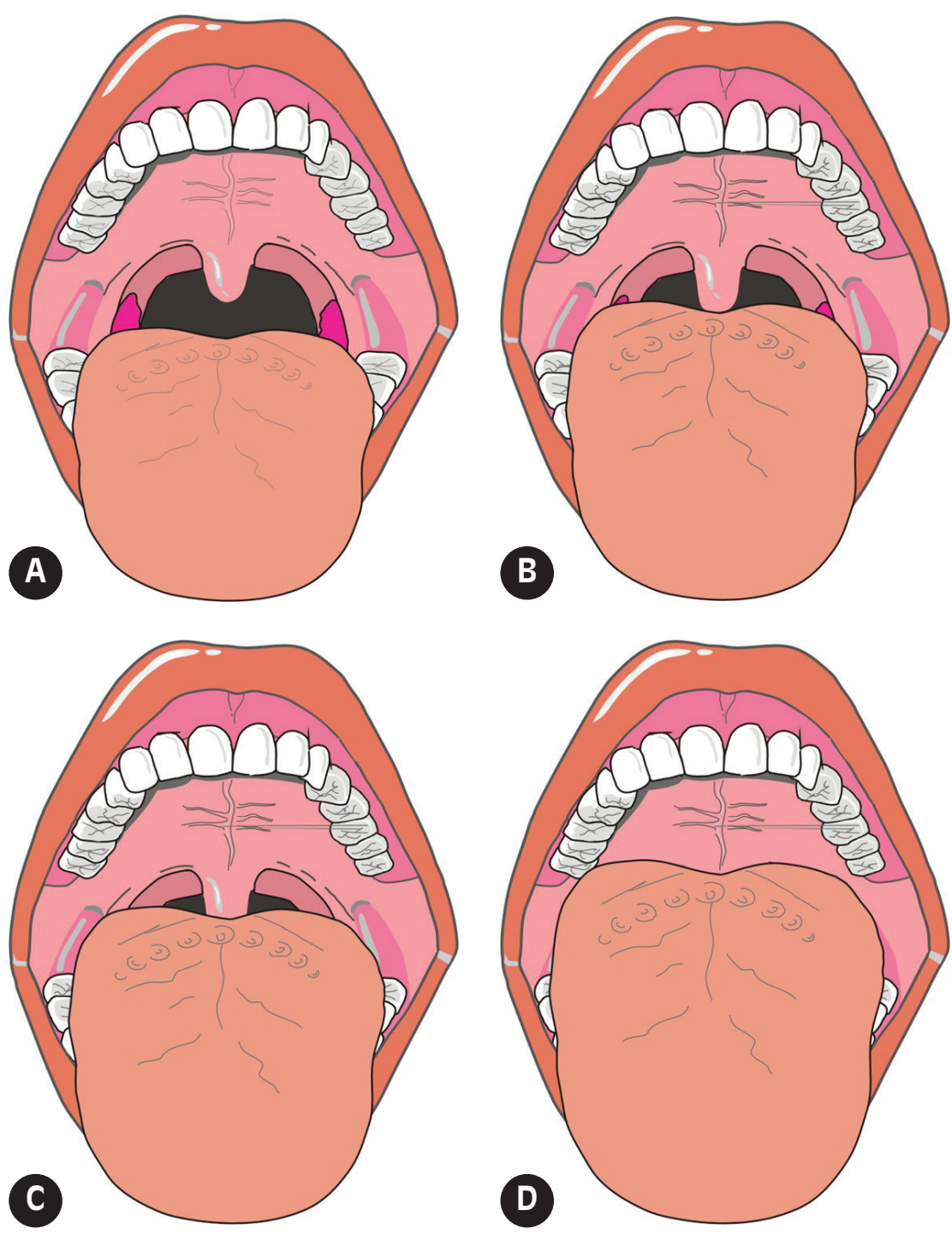

Figura 1: escala de Mallampati-Samsoon. a) grado I. b) grado II. c) grado III. d) grado IV

Merece destacarse que algunos tratamientos precoces de ortopedia funcional de los maxilares, pueden realizar una transformación contraria, aumentando el «espacio vital» de la lengua y además conseguir incrementos del tamaño de la VAS (fundamentalmente a nivel velofaríngeo). Los dispositivos ortopédicos de disyunción palatina (además del aumento turbinal y de válvula nasal anteriormente citados) permiten también incrementar el espacio vital de la lengua.

Desde 1985 hasta nuestros días, se utiliza en anestesiología la puntuación de Mallampati-Samsoon ${ }^{3 *-5}$ con el fin de determinar la dificultad de acceso a la vía aérea durante una intubación endotraqueal. Es un test sencillo que se realiza con el paciente sentado frente al explorador con la boca abierta y la lengua protruida al máximo. Trata de estimar el tamaño de la lengua en relación con la faringe.

Encontramos cuatro tipos de puntuaciones en la escala según las estructuras faríngeas que se visualizan (fig. 1):

I. Paladar blando, istmo de las fauces, úvula, pilares anterior y posterior, amígdala lingual.

II. Paladar blando, istmo de las fauces, úvula

III. Paladar blando, base de la úvula

IV. No se ve el paladar blando

Esta clasificación resulta interesante puesto que se ha relacionado tanto con el riesgo de padecer un SAHOS así como con su gravedad.

Lam y cols 6 $^{6 *}$ y Rombaux y cols? comprobaron cómo las puntuaciones III y IV de la escala de Mallampati podían llegar a tener un valor predictivo en pacientes SAHS del $90 \%$, con una sensibilidad del $91 \%$ y una especificidad del $90 \%$. Cuando las puntuaciones elevadas en dicha escala se asocian a procesos obstructivos nasales, índice de masa corporal elevado y amígdalas aumentadas de tamaño, pasa a tener un valor predictivo muy elevado así como también de la severidad del cuadro obstructivo $0^{8-10^{*}}$.

\section{La Sociedad Americana de Anestesiología}

En 1993 la Sociedad Americana de Anestesiología (ASA) publicó la guía clínica de actuación para establecer una vía aérea difícil para la intuba- 
ción $n^{11^{* *}}$. Con ella pretendía reducir la frecuencia de complicaciones durante las intubaciones (traumatismos de la VAS, daño miocárdico, daño cerebral y/o muerte). En el momento de su publicación el $28 \%$ de las muertes relacionadas con la anestesia estaban relacionadas con la imposibilidad de ventilar con mascarilla o de intubar. También más del $85 \%$ de los juicios por daño cerebral hipóxico o muerte del paciente han sido responsabilidad de problemas en el manejo de la VAS. La intubación endotraqueal es una técnica bien estandarizada pero sin embargo, dependiendo de la experiencia del operador, alteraciones anatómicas o impuestas por la patología, pueden existir situaciones en las que no se puede ventilar al paciente $(0,05-0,35 \%)$. Un 0,01 $2 / 10000$ de los pacientes puede resultar además imposible de intubar. En estos casos si no existen los medios para solucionar la situación rápidamente, el resultado puede ser de muerte o de daño hipóxico severo. Como medida profiláctica de anticipación a estas situaciones se ha buscado identificar signos tales como la desproporción entre integrantes óseos y blandos de la VAS, características cinéticas de la columna cervical y entorno oral, etc. Todo ello permite al anestesista estar advertido para manejar una vía aérea de difícil acceso y además desarrollar protocolos que permiten ahorrar tiempo en los casos de urgencia vital.

\section{Resullados 丩 丩 discusión}

La guía de la Sociedad Americana de Anestesiología ha sido revisada ${ }^{12}$ y

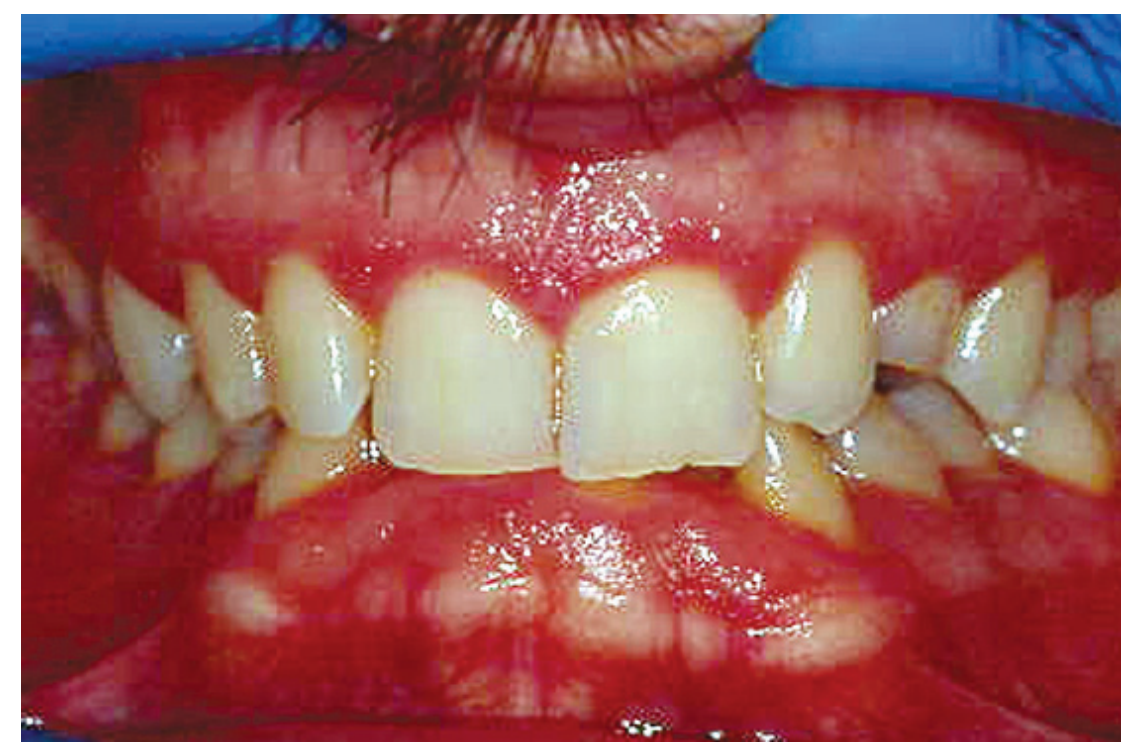

Figura 2: Vista frontal de un paciente con sobremordida profunda.

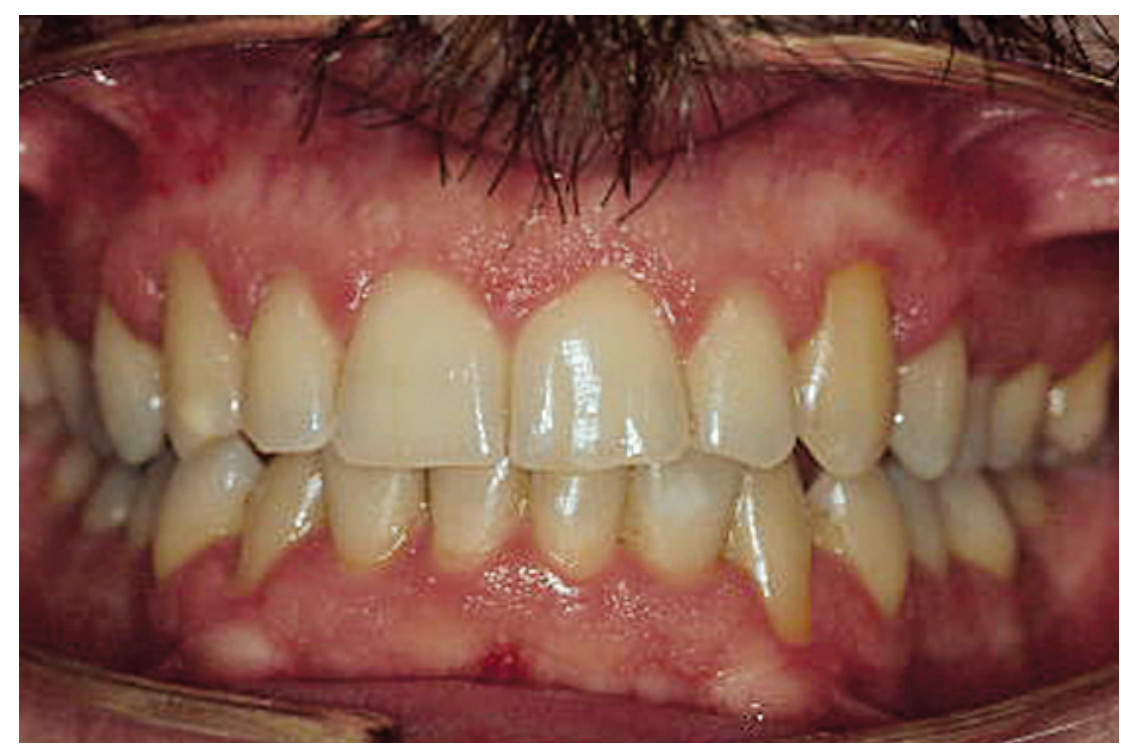

Figura 3: Vista frontal del mismo paciente tras tratamiento ortodóncico.

actualizada ${ }^{13^{* *}}$ por numerosos autores, de ella rescatamos, lo que a criterio de la ASA, se define, como "hallazgos poco tranquilizadores" durante la exploración física previos a la intubación. Trataremos de evidenciar si nuestra disciplina puede llegar a aportar algo ante estas situa- ciones. Con ello pretendemos poner en evidencia la necesidad de dar un enfoque multidisciplinar no sólo ante problemas con patologías médicas complejas (como el SAHS), sino ante exploraciones rutinarias que podrían, incluso reducir la morbimortalidad frente a futuras situa- 


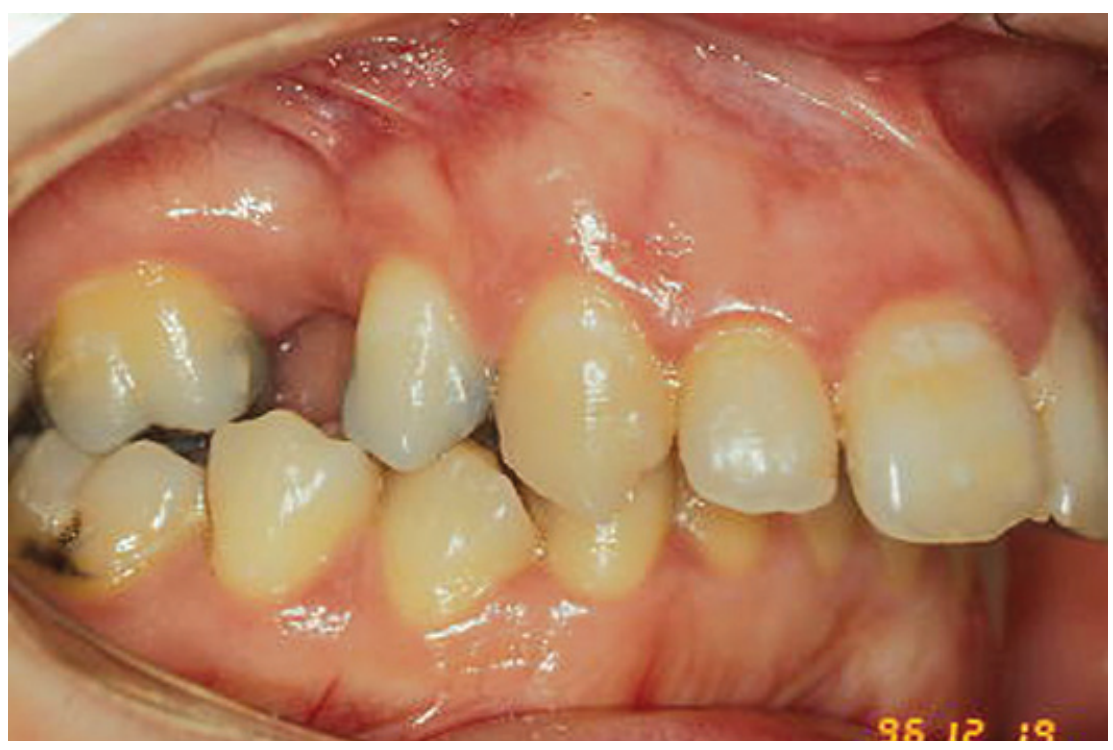

Figura 4: Vista lateral de un paciente con resalte excesivo.

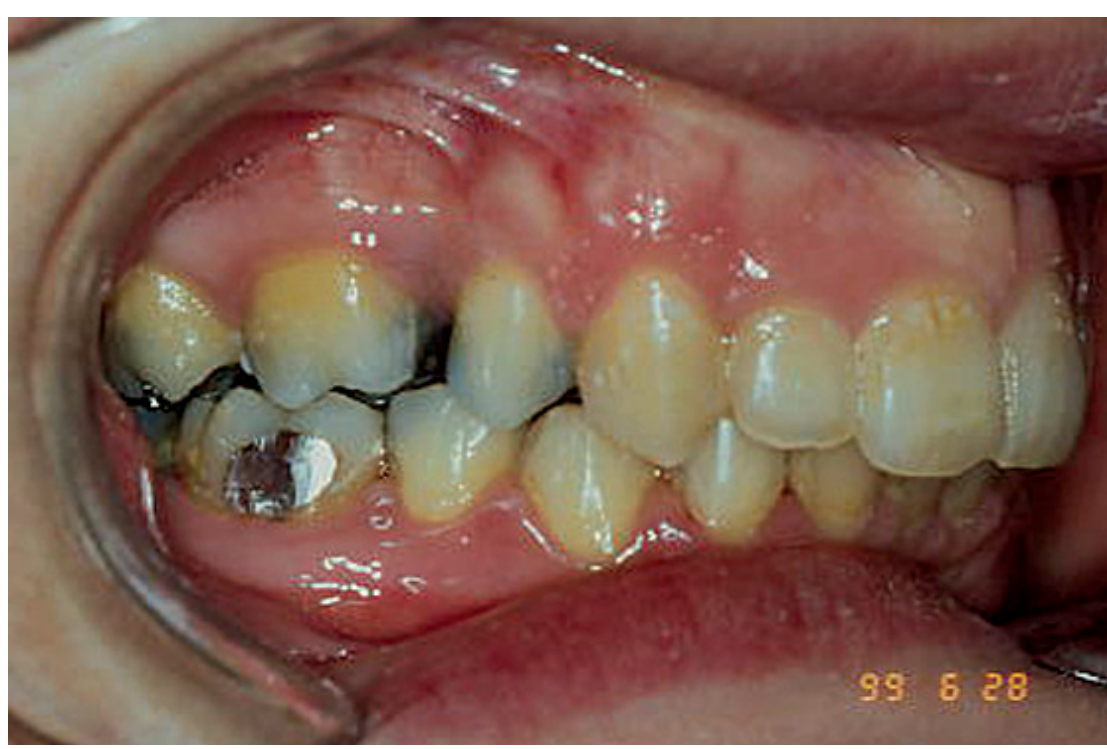

Figura 5: Vista lateral del mismo paciente tras tratamiento ortodóncico.

ciones de riesgo como puede ser una intubación endotraqueal. Desglosaremos a continuación los denominados «hallazgos poco tranquilizadores" de la ASA:

\section{Incisivos superiores largos.}

Si traducimos esto a idioma odon- toestomatológico, nos estamos refiriendo a una situación de sobremordida profunda. Este proceso está reconocido también como una de las causas de fracaso de los DAM en el tratamiento del SAHS.

El paciente en esta situación, para realizar un avance mandibular debe de realizar una rotación mandibular posterior excesiva que:

A) Arrastra al hioides, y estructuras relacionadas con él, hacia atrás «estrangulando» el calibre de la VAS.

B) Limita anatómicamente el avance mandibular (reduce la eficacia de Ios DAM en pacientes SAHS).

Situaciones como la que describimos son habituales en los tratamientos ortodóncicos convencionales (fig. 2) y la resolución de las mismas puede conseguirse en la mayoría de casos de forma satisfactoria (fig. 3)

2. Prominencia de los incisivos superiores respecto de los inferiores durante la aclusión dentaria.

3. El paciente no puede colocar los incisivos inferiores por delante de los superiores durante la protrusión voluntaria mandibular.

Los hallazgos 2 y 3 de la ASA corresponderían, ortodóncicamente hablando, a la presencia de un resalte excesivo que impide o limita una protrusión dentaria eficaz. En última instancia el apartado 3 también podría corresponder con un problema de la articulación temporomandibular, hecho que será tratado en el apartado siguiente. Estos dos hallazgos definen otra de las causas de fracaso o incluso, contraindicación de uso de los DAM en pacientes SAHS. Así la presencia de una protrusiva inferior a $7 \mathrm{~mm}$ desaconseja el uso de DAM en dichos pacientes. Ambos apartados, al igual que el 1, son susceptibles de un tratamiento ortodóncico correctivo, y/o compensador según el caso. (figs. 4 y 5 ). 

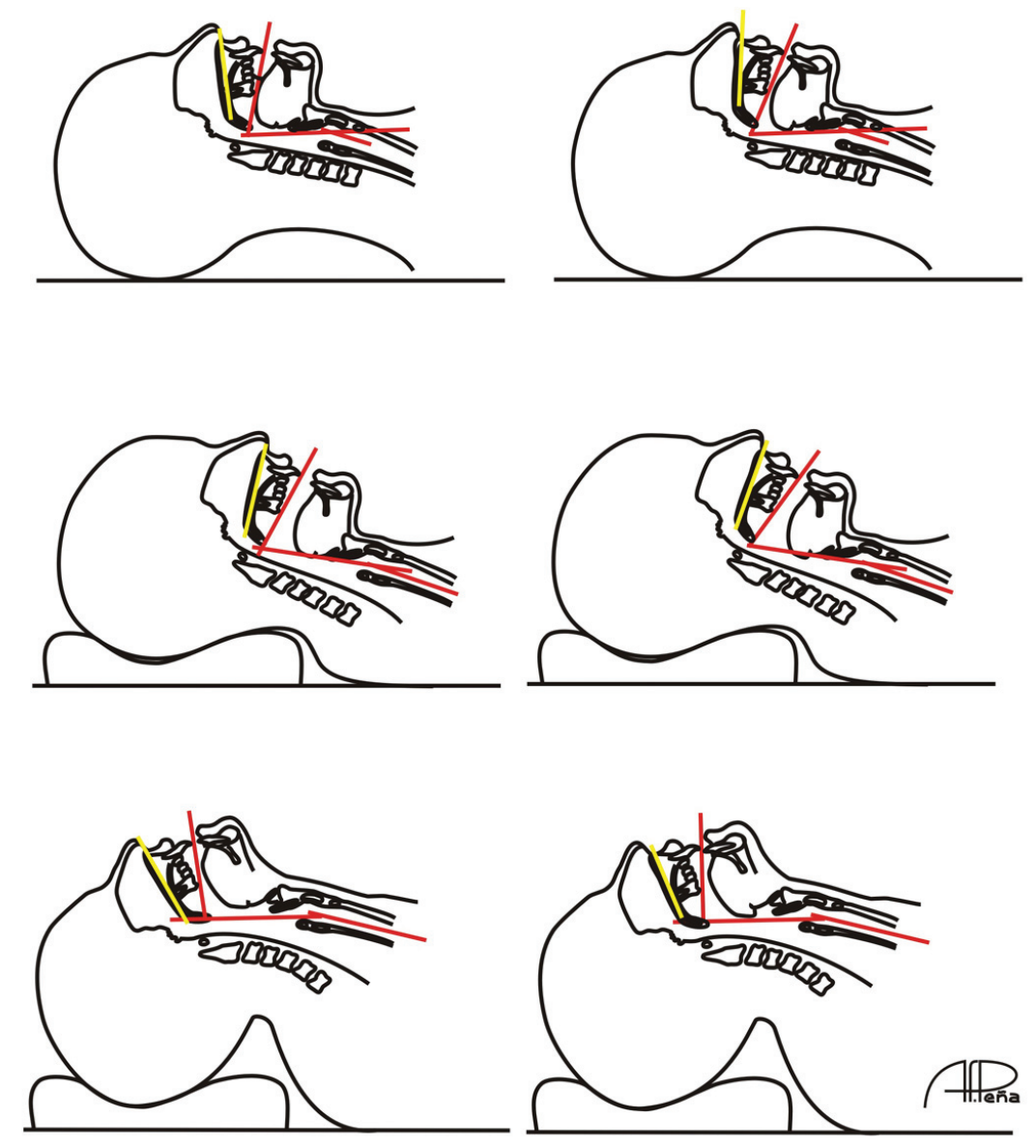

Figura 6: En la imagen ejes bucal, faringeo y laringeo en decúbito, con elevación de $10 \mathrm{~cm}$ y con elevación e hiperextensión en un paciente con normooclusión (izda) y en otro con maloclusión de Clase II, exceso maxilar anterior y sobremordida aumentada [dcha]. Podemos observar el alineamiento relativo de los tres ejes en posición de intubación y la dificultad para hacerlo en el paciente de la derecha.

4. Distancia entre los incisivos superiores e inferiores inferior a 30 $\mathrm{mm}$.

Si hacemos de nuevo la conversión odontológica de esta situación clínica estaremos hablando de una limitación de la apertura mandibular. En condiciones normales la apertura máxima oscila en un valor promedio de 45 $\mathrm{mm}$ desglosados de la siguiente manera: 20-25 mm de movimiento ginglinoide y el resto de movimiento artrodial. Este último es el que suele estar más frecuentemente comprometido en la patología temporomandibular. Los bloqueos articulares van a ser un obstáculo para la laringoscopia por lo que el anestesista se ve obligado a intentar diferenciar si son por alteraciones de la mecánica articular, o por dolor, en cuyo caso puede intentar la maniobra bajo hipnóticos y relajantes musculares. Por otro lado, aunque la etiología de los trastornos de ATM es múltiple (incluso pueden haber sido causados por una intubación previa) ${ }^{14}$, en un gran porcentaje de casos el inicio de la limitación de la apertura está desencadenada por una disfunción de la ATM, que a su vez, puede tener su origen en una maloclusión previamente existente ${ }^{15,16}$.

Una distancia interincisivos inferior a $20 \mathrm{~mm}$ es equivalente a una intubación imposible con laringoscopia convencional. La presencia de una disminución de la apertura es, lógicamente, otra de las limitaciones físicas a la utilización de los DAM para el tratamiento de los problemas respiratorios obstructivos. La intervención odontológico-ortodóncica en el manejo y tratamiento de estos problemas articulares, contribuye, bien a su resolución, bien a la mejora de los mismos. Estos tratamientos podrían aumentar el abanico de posibilidades terapéuticas en pacientes SAHS (haciéndolos candidatos a los DAM) e incluso mejorar las expectativas clínicas de pacientes frente a una posible urgencia vital que precise intubación.

\section{5. Úvula no visible cuando el pa- ciente saca la lengua al máximo en posición sentada.}

Estamos haciendo referencia a los grados III y IV de la escala de Mallampati. Indirectamente podemos relacionar algunas de estas situaciones con limitaciones de la apertura oral, emparentándolos por tanto, con los desórdenes temporomandibulares que hemos tratado previamente. Parte de los supuestos adscritos en este apartado de la ASA quedarían a su vez etiopatogénicamente inmersos en el apartado 


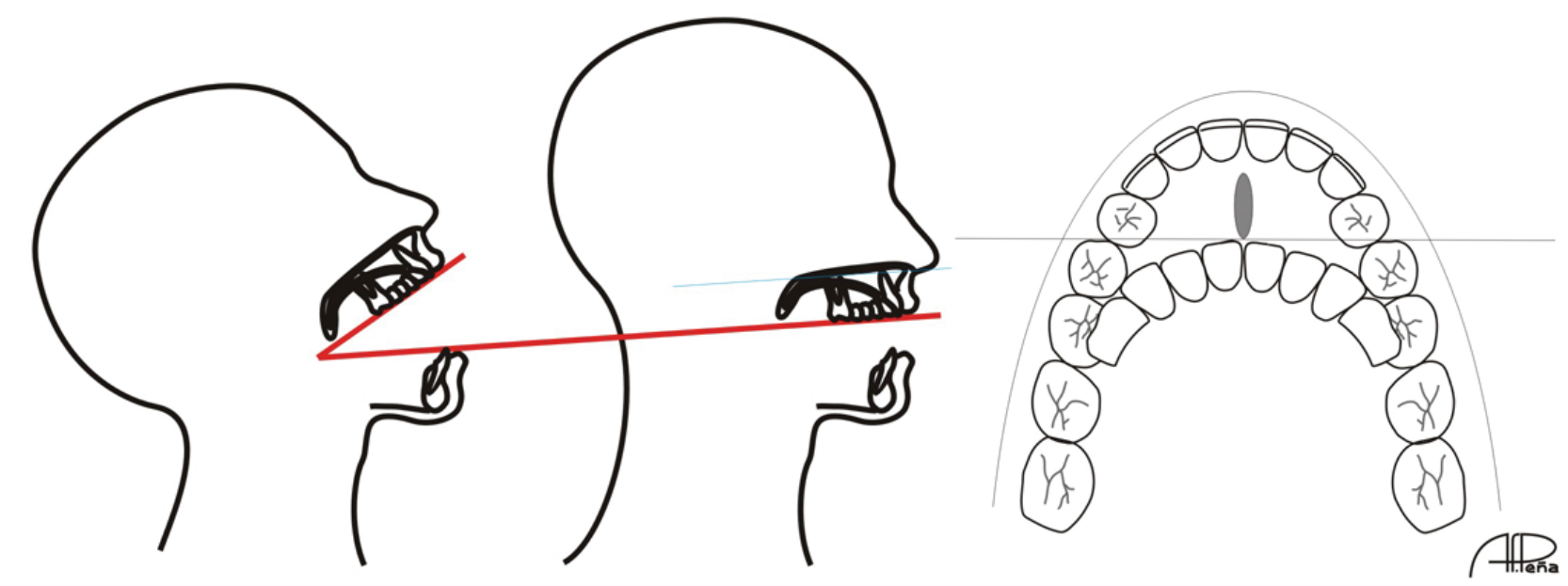

Figura 7: Evaluación clínica de la extensión atlantooccipital y del espacio mandibular anterior en un sujeto normooclusivo.

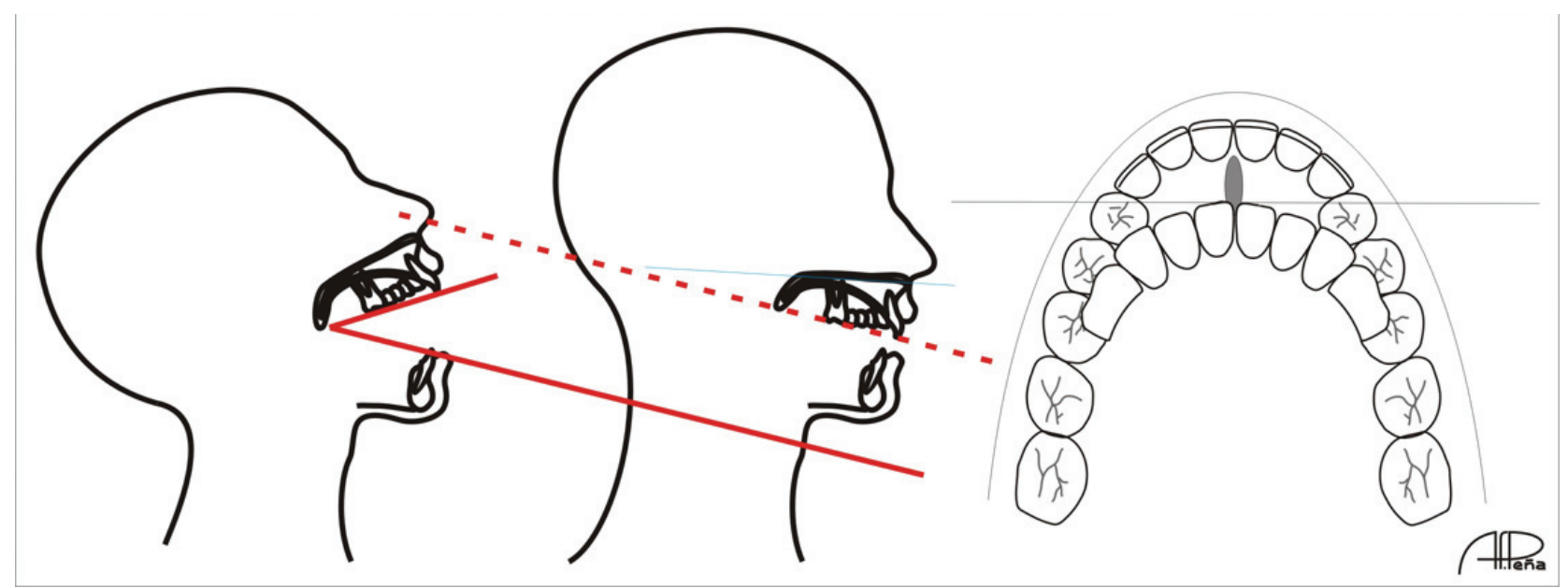

Figura 8: Evaluación clínica de la extensión atlantooccipital y en un paciente con gran sobremordida. Obsérvese cómo se reduce notablemente la longitud del espacio mandibular anterior.

anterior. Esta clasificación pese a las modificaciones que se han propuesto desde su aparición ${ }^{17-19}$ sigue teniendo la misma vigencia junto a un elevado valor predictivo en la identificación de la VAS difícil, máxime cuando se asocia a otros test, como la clasificación de Cormack-Lehane para visualizar la glotis, puntos de Wilson, Bellhouse, Frerk, etc.

Así por ejemplo, de la asociación de las observaciones de Mallampati y
Cormack se estima que cuando el paciente está con la cabeza en posición neutra y podemos ver la úvula y el paladar blando, será altamente probable la visualización clara de la glotis durante la laringoscopia.

\section{Paladar arqueado o estrecho.}

El paladar arqueado o estrecho es uno de los signos característicos de los respiradores orales en los que el desarrollo transversal del maxilar superior está limitado. En estos pacientes la reducción del calibre transversal maxilar contribuye al aumento de las resistencias periféricas caudales y rostrales de las fosas nasales. Nuevamente este apartado, a nuestro juicio, puede ser un condicionante que dificulte la visualización del paladar con lo que su presencia subordinaría la evaluación de la escala de Mallampati. La ortodoncia tiene aquí nuevamente un sitio de privile- 
gio, consiguiendo la corrección del déficit transverso, descenso de la bóveda palatina y un aumento de la distancia interhamular de 3 a $7 \mathrm{~mm}$. Todo ello se acompaña, como dijimos anteriormente, de dilataciones de la válvula nasal y del área turbinal inferior. Estas modificaciones anatómicas mejorarían este apartado y algunos tipos del apartado 5 e incluso podrían contribuir a enderezar un tabique nasal ligeramente desviado.

7. Espacio mandibular rígido, inelástico u ocupado por masas.

\section{Distancia tiromentoniana infe- rior a $6 \mathrm{~cm}$.}

Es la distancia entre la línea media del mentón y la prominencia del cartílago tiroides con el cuello totalmente extendido. Esta medición está estrechamente relacionada con el ángulo atlantooccipital y la longitud del espacio mandibular anterior ${ }^{20}$. Determina la facilidad de alinear los ejes faríngeo y laríngeo. En condiciones normales esta distancia ha de ser superior a 60 $65 \mathrm{~mm}$. Cifras inferiores, indican una posición anterior de la laringe respecto de las estructuras orofaríngeas y dificultad para la exposición glótica. Cuando el ángulo de extensión atlantooccipital (delimitado por el plano oclusal del maxilar superior desde posición neutra hasta la máxima extensión cefálica), es inferior a 30, la visión laringoscópica se ve dificultada. La inclinación del plano oclusal es otro de los elementos que podemos modificar con la aparatología ortopédica funcional. Llegamos a alterar incluso la inclinación de la base del maxilar superior. Esto habitualmente se realiza para compensar las rotaciones que apare- cen con el crecimiento mandibular. Igualmente implicada en este apartado estaría la presencia de cierta hipoplasia mandibular. Aplicando ortopedia funcional podemos minimizar o incluso solucionar este problema, consiguiendo un tamaño mandibular que llegue a lo que McNamara denomina como el «óptimo individual».

Los criterios ASA finalizan con Ios apartados 9, 10 y 11 que corresponden a los epígrafes: cuello corto, cuello ancho, imposibilidad de tocar el tórax con el mentón y dificultad para extender el cuello, hechos estos ante los que desde nuestra perspectiva y como ocurre con los apartados 5 (salvo las excepciones descritas) y 7 no tenemos ninguna función que realizar.

Sabemos que la relación entre el tratamiento ortodóncico y las VAS es estrecha y conocida desde antiguo. Podemos evidenciar cómo el tratamiento con los aparatos funcionales contribuye a incrementar el volumen de la VAS, hecho que se beneficia también de la involución fisiológica adenoidea $2^{21^{* *}, 22^{* * *}}$

Es de sobra conocido que la utilización de DAM durante el sueño reubicando la mandíbula y/o la lengua en una posición más anterior, contribuye a evitar el ronquido y reducir significativamente, en algunos casos, el índice de apneas hipopneas, siendo una de las terapéuticas reconocidas para el tratamiento del SAHS. También se ha comprobado cómo en pacientes dolicocefálicos podemos recurrir a la disyunción maxilar o la cirugía ortognática para incrementar las vía aéreas. En braquicefálicos con sobremordida, la terapéutica electiva para este mismo fin sería un tratamiento ortodóncico previo para corregir primero la sobre- mordida y permitir el posterior avance mandibular.

Conviene recordar que cuando estudiamos la resucitación cardiopulmonar, la primera norma a cumplir es restaurar, y preservar la permeabilidad de la VAS realizando el mismo movimiento que los anestesistas ejercen durante el manejo de la vía aérea como paso previo a una intubación endotraqueal y que no es otro que es una maniobra de desplazamiento anteroinferior de la mandíbula.

Todo ello nos conduce ahora a un hecho claro: de los once criterios de riesgo de la ASA, podemos modificar favorablemente, con tratamientos ortodóncicos y ortopédicos convencionales, seis de ellos y parte de los del apartado 5 .

\section{Conclusiones}

Para concluir nos gustaría hacer hincapié en dos hechos. El primero radica en analizar las posibilidades del tratamiento ortodóncico y la ortopedia dentofacial, ya no como instrumento de tratamiento de situaciones maloclusivas habituales, sino en ir un poco más allá. Nuestros tratamientos pueden beneficiar al paciente como profilaxis de algunas noxas y problemas que, dependiendo del patrón de crecimiento pueden desencadenar patologías importantes en el adulto (léase SAHS) 0 incluso problemas ante situaciones de urgencia, o no, como es la dificultad de intubación previa a una intervención quirúrgica. Si tomamos conciencia de que un elevado porcentaje de la población requerirá una intubación al menos una vez en la vida podremos hacer- 
nos una idea de la dimensión de esta afirmación.

El segundo vendría a avalar la necesidad de interrelación entre los especialistas que nos dedicamos a la salud y que nos movemos en territorios comunes, ya que como acaba mos de ver, algo que puede parecer tan banal como la posición de los dientes puede llegar a tener una trascendencia mayor de la que a veces la población sanitaria en concreto y los pacientes en general creen.

\section{Agradecimientos}

A la Dra. María José Navarro Royo del Complejo Hospitalario San MillánSan Pedro de la Rioja y al Dr. Jesús Moreno Badiola profesor del Máster de periodoncia de la UCM por su colaboración desinteresada en la elaboración de este artículo.

\section{Bibliografia recomendada}

Para profundizar en la lectura de este tema, el/los autor/es considera/an interesantes los artículos que aparecen señalados del siguiente modo: *de interés ${ }^{* *}$ de especial interés.

1*. Timms DJ. Rapid Maxillary expansion in the treatment of nocturnal enuresis. Angle Orthod 1998;60(3):229-33.

Efectos de la expansión maxilar rápida en la vía aérea superior y su implicación en la resolución de patologías asociadas (enuresis).

2*. Kurol J. Othodontic maxillary expansion and its effect on nocturnal enuresis. Angle Orthod 1998;68(3):225-30.

Efectos de la expansión maxilar rápida en la vía aérea superior y su implicación en la resolución de patologías asociadas (enuresis)

$3^{* *}$. Mallampati SR, Gatt SP, Gugino LD y cols. A Clinical sign to predict difficult tracheal intubation: a prospective study. Can Anaesth Soc J. 1985;32(4):429-34.

Estudio base sobre la exploración intraoral y sus inplicaciones en la intubación de los pacientes. Su uso se estandarizado en la mayoría de los Servicios de anestesia en todo el mundo.

4. Samsoon GL, Young JR. Difficult tracheal intubation: a retrospective study. Anaesthesia 1987;42:487-90.

5. Iohom G, Ronayne M, Cunningham AJ. Prediction of difficult tracheal intubation. Eur $\mathrm{J}$ Anaesthesiol 2003;20(1):31-6.

6**. Lam B, Ryan F, Lam WK. Mallampati score is a good and independent predictive factor for obstructive sleep apnoea. Medical Research Meeting, University Department of Medicine, The University of Hong Kong, Hong Kong Practitioner 2000;22(Supp 2):18. Papel de las anomalías anatómicas intraorales como factores de riesgo del síndrome de apnea del sueño

7. Rombaux P, Bertrand B, Boudewyns A y cols. Standard ENT clinical evaluation of the sleep-disordered breathing patient; a consensus report. Acta Otorhinolaryngol Belg 2002;56 (2):127-37
8. Liistro G, Rombaux P, Belge C, Dury M, Aubert G, Rodenstein DO. High Mallampati score and nasal obstruction are associated risk factors for obstructive sleep apnoea. Eur Respir J 2003;21(2):248-52.

9. Erdamar B, Suoglu Y, Cuhadaroglu C y cols. Evaluation of clinical parameters in patients with obstructive sleep apnea and possible correlation with the severity of the disease. Eur Arch Otorhinolaryngol 2001;258(9):492-5.

10*. Friedman M, Tanyeri H, La Rosa M y cols. Clinical predictors of obstructive sleep apnea. Laryngoscope 1999;109(12):1901-7.

Descripción de los signos clínicos considerados como factores de riesgo de padecer apnea del sueño.

11**. Caplan RA, Bneumof JL, Berry FA y cols. Practice guidelines for the maagement of difficult airway. A report by the merican Society of anesthesiologists Task Force on the Management of the difficult airway. Anesthesiology 1993;78:597-602.

Descripción de los parámetros clínicos que pueden ayudar a identificar casos específicos de dificultades en la intubación y toma de decisiones ante los mismos.

12. Aronson WL, McAuliffe MS, Miller K. Variability in the American Society of Anestesiologists Phisical status Classification scale. AANA J 2003;71(4):265-74

13**. Caplan RA, Bneumof JL, Berry FA y cols. Practice guidelines for the management of the difficult airway. An updated report by the American Society of Anesthesiologists Task Force on the Management of the Difficult Airway. Anesthesiology 2003;98:1269-77. Actualización y ampliación de los supuestos descritos en el año 1993 por la ASA.

14. Lipp M, Daublander M, Thierbach A, Reuss U. Movement of temporomandibular joint during tracheal intubation. Anaesthesist 1996;45(10):907-22.

15. Brown DT, Gaudet EL Jr. Temporomandibular disorder treatment outcomes:second report of a large scale prospective clinical study. Cranio 2002;20(4):224-53.

16. Carlsson GE, Egermark I, Magnusson T. Predictors of bruxism, others oral parafunctionals, and tooth wear over a 20-year follow-up period. J Orofac Pain 2003;17:50-7.

17. Khan ZH, Kashfi A, Ebrahimkhani E. A comparison of the upper lip bite test (a simple new technique) with modified Mallampati classification in predicting difficulty in endotracheal intubation: a prospective blinded study. Anesth Analg 2003;97(3):9156 .

18. Sinha PK, Mitra S, Gombar S, Gombar KK. A new class for modified Mallampati sign. Anesth Analg 1999;89(1):259-60.

19. Krishnakar MV. Reliability of Mallampati classification in upper airway pathology. Anaesthesia 2001;56(8):804.

20. Bellhouse CP, Doré C. Criteria for estimating likehood of difficulty of endotracheal intubation with the Macintosh laryngoscope. Anaesth Intensive Care 1988;16:329-37.

21**. Özbek MM, Memiklogu UT, Gögen H, Lowe A. Oropharingeal airway dimensions and functional-orthopedic treatment in skeletal Class II cases. Angle Orthod 1998;68(4):327-36. Implicaciones del tratamiento ortodóncico en la vía aérea superior.

22**. Cobo J. El Activador y las Vías Aéreas Superiores. Rev Esp Ortod 1994;24:311-8. Trabajo pionero en el tema de las vías aéreas donde se muestra cómo un aparato funcional de tratamiento ortopédico desencadena un incremento de la VAS clínica y estadísticamente significativo. 